\title{
The economics of managing tuberculosis in cancer patients in an oncology center in eastern India
}

\author{
Zoe Bennett ${ }^{1}$, Raja Dhar MD, MRCP, FCCP, MSc ${ }^{2}$, Kingshuk Dhar MD ${ }^{3}$, Krishnendu Das BSc, DMLT ${ }^{3}$, \\ Anusha Harishankar MS ${ }^{3}$, Sanjay Bhattacharya MD, DNB, DipRCPath, FRCPath ${ }^{3}$ and \\ Mammen Chandy MD, FRACP, FRCPA, FRCP \\ ${ }^{1}$ London School of Economics, London, United Kingdom, ${ }^{2}$ Department of Pulmonology, Tata Medical Center, Kolkata, India, ${ }^{3}$ Department of Microbiology, Tata \\ Medical Center, Kolkata, India and ${ }^{4}$ Department of Clinical Hematology, Tata Medical Center, Kolkata, India
}

Table 1. Details of Cost Analysis in Treatment of Tuberculosis

To the Editor-Cancer increases the chance of tuberculosis because of decreased immunity from disease and treatment (eg, chemotherapy). An intermediate tuberculosis (TB) prevalence country (South Korea) reported a cancer-specific standardized incidence ratio (SIR) for TB of 2.22 among all cancer patients, with the highest rate observed in those with hematologic malignancies (SIR, 6.67) and lowest in those with biliary tract cancers (SIR, 1.34). ${ }^{1}$ Understanding the problem of TB in the context of cancer is important because (1) cancer may develop in the background of a previous TB infection; (2) TB may exist concurrently with malignancy in the same patient or clinical specimen; and (3) many diagnostic challenges arise from the multifaceted presentations of these 2 disorders. ${ }^{2}$ Furthermore, TB leads to direct costs for diagnosis, treatment, and admission, as well as indirect costs from loss of productivity and other societal costs. It has been estimated that $42 \%$ of the households with TB patients incurred catastrophic health expenditures from hospitalization due to TB in India compared to $79 \%$ for households with cancer patients in 2014. In addition, the likelihood of incurring financial distress was 3.2 times higher for those hospitalized for cancer and 2.6 times for TB patients. ${ }^{3}$

This retrospective study was undertaken in a cancer hospital in eastern India between June 2011 and May 2016 among all patients with culture-confirmed tuberculosis to evaluate the direct healthcare costs attributable to TB in cancer patients. The period chosen to calculate costs spanned from 90 days prior to the TB diagnosis to 1 year after the diagnosis to limit the research primarily to the costs surrounding $\mathrm{TB}$, rather than the costs incurred due to cancer. In total, we identified 118 patients with culture-confirmed TB during the study period of 60 months ( 2 patients per month). The median age of the TB patients was 48.2 years (range, 6.3-82.1 years); 63 were women (53\%) and 55 were men (47\%). Of 118 patients, 45 (38\%) had a hospital admission during the stipulated follow-up duration for either investigation or treatment. The number of deaths within this period was 4 (3.4\%), with 3 deaths occurring in the first 30 days, no deaths in the 90-day period, and 1 death within 1 year of TB confirmation. The mean duration of follow-up of the patients after $\mathrm{TB}$ culture positivity was 204 days (6.8 months).

The average cost per patient was Indian rupees (Rs.) 19,295 (US\$266; 46.1\%) for investigation, Rs. 18,686 (US\$258; 44.7\%) for

Author for correspondence: Dr Sanjay Bhattacharya, Tata Medical Center, 14 Major Arterial Road (E-W), New Town, Kolkata 700 160, India. E-mail: drsanjay1970@hotmail. com

Cite this article: Bennett Z, et al. (2019) The economics of managing tuberculosis in cancer patients in an oncology center in eastern India. Infection Control \& Hospital Epidemiology 2019, 40, 122-124. doi: 10.1017/ice.2018.284

\begin{tabular}{lcc} 
& \multicolumn{2}{c}{ Cost } \\
\cline { 2 - 3 } Type of Tuberculosis & Rs. USD \\
\hline Pulmonary TB & 1,140 & 15.72 \\
\hline Intensive phase & 1,020 & 14.07 \\
\hline Continuation phase & 2,448 & 33.76 \\
\hline Total (includes vitamin B6) & & 15.72 \\
\hline Extra-pulmonary TB & 1,140 & 35.17 \\
\hline Intensive phase & 2,550 & 58.83 \\
\hline Continuation phase & 4,266 & $1,035.11$ \\
\hline Total (includes vitamin B6) & $183,420-237,600$ & $2,529.45-3,276.62$ \\
\hline MDR-TB & 75,060 & $1,494.34-2,241.51$ \\
\hline 5 drugs, 6 mo & & \\
\hline 4 drugs, 12-18 mo & & \\
\hline Total & & \\
\hline
\end{tabular}

Note. Rs., Indian rupees; TB, tuberculosis; MDR, multidrug-resistant.

admission cost, and Rs. 3,864 (US $\$ 53 ; 9.2 \%$ ) for consultation fees. The investigation costs were divided into 2 categories: radiology (Rs. 10,383, US\$143) and laboratory tests (Rs. 8,912, US\$123). The laboratory costs were divided into 3 subcategories: hematopathology laboratory (Rs. 2,397, US\$33), biochemistry (Rs. 3,613, US\$50), and mycobacteriology (Rs. 2,902, US\$40). When all costs were considered, the total average cost for 1 patient within the 15-month period was Rs. 41,846 (US\$577 without anti-TB drugs and \$636 with anti-TB drugs) (Tables 1 and 2 and Supplementary Fig. 1).

Tuberculosis treatment drains healthcare and social resources. From the provider perspective, the mean drug-sensitive-TB treatment cost per patient varies among countries depending on the state of the economy, from US\$14,659 in high-income countries to US $\$ 258$ in low-income countries. The costs for treating multidrug-resistant (MDR)-TB ranges from US $\$ 83,365$ in high-income countries to US $\$ 1218$ in low-income countries. Productivity losses (calculated based on time lost due to seeking treatment and being ill; and taking into account country income) from TB have been estimated to be $16 \%$ in high-income countries and $38 \%$ in low-income countries. ${ }^{4}$ A study from rural India reported that the mean direct cost per patient due to TB was US 
Table 2. Doses and Regimens for Drug-Sensitive and Drug-Resistant Tuberculosis and Cost of Treatment

\begin{tabular}{|c|c|c|}
\hline \multirow[b]{2}{*}{ Regimen and Dose } & \multicolumn{2}{|c|}{ Cost } \\
\hline & Rs. & USD \\
\hline \multicolumn{3}{|l|}{ Drug-Sensitive $\mathrm{TB}^{\mathrm{a}}$} \\
\hline \multicolumn{3}{|l|}{ Pulmonary: 6-mo therapy } \\
\hline 4 drugs for 2 mo (INH, rifampicin, ethambutol, pyrazinamide) & 1,140 & 15.72 \\
\hline 2 drugs for 4 mo (INH, rifampicin) & 1,020 & 14.07 \\
\hline vitamin $\mathrm{B} 6,6 \mathrm{mo}$ & 288 & 3.97 \\
\hline Total & 2,448 & 33.76 \\
\hline \multicolumn{3}{|l|}{ Extra-pulmonary: 12-mo therapy } \\
\hline 4 drugs, 2 mo (INH, rifampicin, ethambutol, pyrazinamide) & 1,140 & 15.72 \\
\hline 2 drugs, 10 mo (INH, rifampicin) & 2,550 & 35.17 \\
\hline vitamin $\mathrm{B} 6,12 \mathrm{mo}$ & 576 & 7.94 \\
\hline Total & 4,266 & 58.83 \\
\hline \multicolumn{3}{|l|}{ MDR-TB } \\
\hline Amikacin, 750 mg IV Q24H & 116 & 1.60 \\
\hline Moxifloxacin, $400 \mathrm{mg}$ PO Q24H & 27 & 0.37 \\
\hline Pyrazinamide, $1,000 \mathrm{mg}$ PO Q24H & 10 & 0.14 \\
\hline Ethionamide, 1,000 mg PO Q24H & 64 & 0.88 \\
\hline Cycloserine, 1,000 mg PO Q24H & 200 & 2.76 \\
\hline All 5 drugs, 6 mo & 75,060 & $1,035.11$ \\
\hline 4 drugs (amikacin IV excluded), $12-18$ mo & $108,360-162,540$ & $1,494.34-2,241.51$ \\
\hline Total & $183,420-237,600$ & $2,529.45-3,276.62$ \\
\hline
\end{tabular}

Note. Rs., Indian rupees; TB, tuberculosis; MDR, multidrug-resistant; IV, intravenous; PO, per oral; Q24H, per 24-hour period.

${ }^{a}$ Adult standard dose: INH, $300 \mathrm{mg}$ PO Q24H ( $\left.5 \mathrm{mg} / \mathrm{kg}\right)$; rifampicin, $600 \mathrm{mg}$ PO Q24H (<50 kg: $450 \mathrm{mg}$ PO Q24H) $(10 \mathrm{mg} / \mathrm{kg}) ; \mathrm{pyrazinamide,} 2000 \mathrm{mg}$ PO Q24H (<50 kg: $1500 \mathrm{mg}$ PO Q24H) $(15-30 \mathrm{mg} / \mathrm{kg})$; ethambutol, $1200 \mathrm{mg}$ PO Q24H (<55 kg: $800 \mathrm{mg}$ PO Q24H) $(15 \mathrm{mg} / \mathrm{kg})$.

$\$ 35$, the mean indirect cost per patient was US\$527, and the total mean cost per patient was US $\$ 563 .^{5}$ Of the smear-positive TB patients, $25 \%$ had to be admitted to the hospital, at a mean cost of $\$ 279$ per admission. ${ }^{5}$ Our study showed a higher admission rate (38\%) but a similar admission cost compared to the southern Indian study, despite the fact that our cohort had sicker patients (due to cancer-related comorbidities) and that our hospital is a not-for-profit private trust. In a Turkish series of 16 cases of TB with cancer, 5 patients (31.3\%) died during treatment, a mortality rate significantly greater than in our cohort $(3.4 \%)^{6}$

The mean total spending on hospitalization for tuberculosis has been estimated to be Rs. 13,121 (US\$202) in India, with $99.9 \%$ being out-of-pocket expenditure by patients. ${ }^{7}$ However, our study revealed a higher expenditure (US\$258). In India, for cancer treatment, the total mean cost of hospitalization was reported to be Rs. 62,349 ( $\$ 860$ ), but $8.2 \%$ reimbursement was received from the government. ${ }^{7}$ In an Indian study from Tamil Nadu on patients with pulmonary tuberculosis, patients experienced a median of 6 days of time to treatment initiation and 21 days of health system delay. ${ }^{8}$ Between 2012 and 2016, the mycobacteriology workload in our hospital increased from 258 to 651 samples (an increase of 152\%), and new patient registrations increased from 10,606 to 15,479 (an increase of 45\%). In parallel, the hospitalization rate in India due to tuberculosis increased by $24 \%$ (all ages combined) from 1995 to 2014, whereas for cancer a $216 \%$ increase occurred during the same period. ${ }^{7}$ In conclusion, the current study provides insight regarding the cost of $\mathrm{TB}$ management under the wider spectrum of cancer care. Hospitalization rates due to TB and cancer in India are 50 and 87 per 100,000 population, respectively, and $\mathrm{TB}$ continues to be an important infection in oncology patients. The economic impact of $\mathrm{TB}$ needs to be factored in while planning healthcare resource requirements of patients with cancer.

Acknowledgments. This study was conducted as part of the Tata Social Internship Program.

Financial support. No financial support was provided relevant to this article.

Conflicts of interest. All authors report no conflicts of interest relevant to this article.

Supplementary materials. To view supplementary material for this article, please visit https://doi.org/10.1017/ice.2018.284 


\section{References}

1. Seo GH, Kim MJ, Seo S, et al. Cancer-specific incidence rates of tuberculosis: a 5-year nationwide population-based study in a country with an intermediate tuberculosis burden. Medicine (Baltimore) 2016;95:1-5.

2. Falagas ME, Kouranos VD, Athanassa Z, Kopterides P. Tuberculosis and malignancy. QJM 2010;103:461-487.

3. Kastor A, Mohanty SK Disease-specific out-of-pocket and catastrophic health expenditure on hospitalization in India: Do Indian households face distress health financing? PLoS One 2018;13:1-18.

4. Laurence YV, Griffiths UK, Vassall A Costs to health services and the patient of treating tuberculosis: a systematic literature review. Pharmacoeconomics 2015;33:939-955.
5. John KR, Daley P, Kincler N, Oxlade O, Menzies D Costs incurred by patients with pulmonary tuberculosis in rural India. Int $J$ Tuberc Lung Dis 2009;13;1281-1287.

6. Çakar B, Çiledağ A Evaluation of coexistence of cancer and active tuberculosis; 16 case series. Respir Med Case Rep 2017;23:33-37.

7. Kastor A, Mohanty SK Disease and age pattern of hospitalisation and associated costs in India: 1995-2014. BMJ Open 2018;8:1-12.

8. Veesa KS, John KR, Moonan PK, et al. Diagnostic pathways and direct medical costs incurred by new adult pulmonary tuberculosis patients prior to anti-tuberculosis treatment, Tamil Nadu, India. PLoS One 2018;13:1-14. 